\title{
Comparison of distal versus proximal-distal kinesio taping effects on gait initiation profile in athletes with functional ankle instability
}

\author{
S. Fereydounnia ${ }^{1}$, A. Shadmehr ${ }^{1}$, B. Attarbashi Moghadam¹, \\ S. Talebian Moghadam¹, S. Mohsen Mir ${ }^{1}$, S. Salemi², F. Pourkazemi ${ }^{3}$ \\ 1 Physical Therapy Department, School of Rehabilitation, Tehran University of Medical Sciences, Tehran, Iran \\ 2 Rehabilitation Faculty, Shiraz University of Medical Sciences, Shiraz, Iran \\ 3 Physical Therapy Department, The University of Sydney, Sydney, Australia
}

\section{CORRESPONDING AUTHOR:}

Azadeh Shadmehr

School of Rehabilitation

Tehran University of Medical Sciences

PicheShemiran, Enghelab Street

Tehran, Iran

E-mail: shadmehr@tums.ac.ir

Tel: 0098- 21- 77528468

DOI:

$10.32098 / \mathrm{mltj} .02 .2019 .14$

LEVEL OF EVIDENCE:

$1 \mathrm{~b}$

\begin{abstract}
SUMMARY
Objective. The objectives of this study were to investigate the impact of two application methods of kinesio taping on gait initiation (GI) profile of athletes with and without functional ankle instability (FAI): 1) distal (applied on the peroneus longus muscle), 2) proximal- distal (applied on gluteus medius and peroneus longus muscles). Methods. Experiment consisted of a test- retest study of the immediate - term effects of the two methods of kinesio taping in two separate weeks on GI profile in 15 athletes with FAI and 15 healthy athletes. Participants performed bare feet GI on a force plate which captured center of pressure (COP). The GI profile was separated into three phases (anticipatory, weight transfer, and locomotive) and two directions (anteroposterior, mediolateral). The averages of 3 trials were used for analyses. Multifactorial repeated measure ANOVA were used for comparison of the two application methods and both group of participants. Results. There weren't any significant result for factor effect and group effect for all of the 9 variables of the study $(\mathrm{P}>0.05)$. Also, we did not observe any significant group by factor interaction effects $(\mathrm{P}>0.05)$, indicating that each group respond to the interventions in a similar pattern. Conclusion. The distal or proximal- distal application of kinesio taping had no impact on GI profile of athletes with and without FAI. We concluded that it is better to assess the long- term effects of kinesio taping in the ore challenging environment and tasks.
\end{abstract}

\section{KEY WORDS}

Functional ankle instability; gait initiation profile; center of pressure; kinesio taping; facilitation technique

\section{INTRODUCTION}

Lateral ankle sprains are one of the most common injuries of musculoskeletal system (1). Sprains and strains as one of the most common out-patient problems in the United States, causing 4.4 million referrals to emergency departments per year (2). Evidence has demonstrated that athletes are twice more likely to sustain an ankle sprain the year after injury. Nearly 50\% of recurrent sprains leads to chronic pain and instability and potentially causes long-term disability and long-term medical care (3). The high rate of sprains among all the sports and consequently its negative consequences such as participa- tion limitation in the tournaments, necessitates preventive approaches (3).

In our previous studies, participants with chronic ankle instability (CAI) demonstrated delayed muscle activation, reduced strength and contraction duration in their peroneus longus $(4,5)$. A number of studies have shown that patients with functional ankle instability (FAI) are more dependent on the hip strategy rather than ankle strategy. The ankle strategy includes the shift in the center of mass (COM) with the trunk pivoting on the ankle joint, while the hip strategy involves the use of gluteus medius muscle for postural modification (6). Individuals with a chronic ankle sprain have a 
weaker hip abductors and have less plantarflexion ROM in their injured side. Hip stability and strength are important for proper foot positioning during heel strike. Because of the complex and continuous nature of the lower limb kinematics, it is very important to consider the all lower limb joints for walking stability (7). Therefore, in designing a therapeutic program for the rehabilitation of patients with ankle sprain, improving the muscle function should be considered, especially peroneus longus and gluteus medius muscles.

On the other hand, the postural control is clearly impaired after acute ankle sprain, and it is visible on both injured and non- injured legs in comparison with control. Although it seems that the FAI is also associated with the modified postural control, but these defects were not detected using traditional measurements of COP (8). Recently, researchers looked at the reliability of gait initiation (GI) profile in the three separate anticipatory, weight transfer, and locomotive phases in individuals with chronic ankle instability, and suggested that a high reliability of GI (ICC $\geq 0.76$ ) could be used to evaluate patients with CAI and also it is important to determine changing GI profile in these individuals after different types of interventions (9). It seems that GI profile could be detected the neuromuscular adaptations following FAI and relevant interventions.

Despite advances in prevention protocols for improving the strength, flexibility, proprioception and neuromuscular control, ankle sprain are still highly prevalent in athletes (10). Recently, kinesio taping has been used to reduce pain and inflammation and is claimed to improve joint position sense, joint stability, muscle strength, functional performance and balance (11), however, there are discrepancies in findings of studies. For a professional athlete, a small improvement in performance can mean a difference between a gold medal and a silver medal. Sport activities that require jumping, shooting and throwing, and a sudden burst of power to change direction, require an explosive muscle power.

It is possible that the use of kinesio taping can improve the explosive power by increasing sensory input to the neuromuscular system. Thus, kinesio taping is a modality that has the potential to improve athletic performance, which depends on the muscle's explosive power. It has been shown to improve muscle tone through the recruitment of muscle spindles. Also, it stimulates the mechanical receptors in skin and, by reflex action causes changes in the muscle tone (12). The present study intends to investigate the immediateterm effects of two different applications of kinesio taping: 1) Distal (over peroneus longus), 2) Proximal- Distal (over gluteus medius- peroneus longus) on GI profile in athletes with and without functional instability. We hypothesize that the kinesio taping application can change the neuromuscular characteristics by its effects on GI profiles.

\section{METHODS AND MATERIAL}

This study was approved by Ethics Committee at the Tehran University of Medical Sciences (IR.TUMS.MEDICINE. REC.1396.4225) and Iranian Registry of Clinical Trials (IRCT20171028037057N1). The study was performed in the Biomechanic Laboratory of the School of Rehabilitation, Tehran University of Medical Sciences.

\section{PARTICIPANTS}

Participants were recruited from sport clubs via announcement. To be included, participants' age was ranged between 18 to 32 year old. Participants were included in the FAI group if they reported a history of at least one ankle sprain on one side in the past 6 months to 1 year and at least two episodes of a "giving way" sensation. They also had to report decreased function due to their history of ankle sprains by scoring $90 \%$ on the Foot and Ankle Ability Measure (FAAM) and $80 \%$ on the FAAM-Sport (for FAI group) (13). Participants were included in the control group if they had no history of ankle sprains or no feeling of ankle joint instability on either side. All the participants exercised regularly; three sessions a week and each session was at least for $2 \mathrm{~h}$.

Participants were excluded if they had a history of lower extremity surgery or fracture, balance impairments or neurological disorders, skin allergy to kinesio taping.

\section{STUDY PROCEDURE}

Prior to commencement of the study, the examiner explained the objectives and the procedure of the study to the participants. After signing the consent form, participants completed the initial assessment by providing their demographic data via a questionnaire and measurements of their weight and height. Participants completed two intervention sessions with a seven-day interval. Participants were instructed to maintain normal activities between the sessions. The order of interventions was randomised during the first session, using concealed opaque envelops with a red or blue card inside. The red card was allocated to the distal application of kinesio taping and the blue card was allocated to the proximal- diatal application of the kinesio tape on the first sessions. In the following week, the intervention method was the opposite of the first week. The outcome measures were collected on both occasions, before and 30 minutes after the interventions.

\section{INTERVENTION}

At the beginning of each session, the examiner asked the participants to do the stretching and warm-up exercises for lower extremities to prevent the possible injury during the 
test. A certified Kinesio taping practitioner administered all treatments. The kinesiology tape (Red Kinesio Tex Tape, Tmax, South Korea) was used for all interventions. For participants with FAI, the kinesio taping was applied on the affected side. The tested side in the control group was matched with the participants with FAI.

In the distal application of kinesio taping, muscle facilitation technique was used for increasing the tone of peroneus longus muscle. The participants were in the long sitting position. The anchor of the I-strip was attached to the head of fibula without tension. The base was attached to the pathway of peroneus longus with $35 \%$ tension and at the same time therapist asked the participants to do the plantar flexion and inversion in order to apply stretch on the tissue. The end of the kinsio tape was attached to the plantar surface of the first metatarsal bone(figure 2, A)(16).

In the proximal- distal application of kinesio taping, in addition to the peroneus longus facilitation technique, the gluteus medius facilitation technique was used. The first third of one I-strip began at the posterior iliac crest without tension to provide an anchor that did not cross the target tissue. The participants actively flexed the adducted hip to allow application of the middle third of the tape with approximately $35 \%$ tension (able to visualize the wave pattern in the tape). Subsequently, with the leg in the original position, the remainder of the tape was applied without tension,

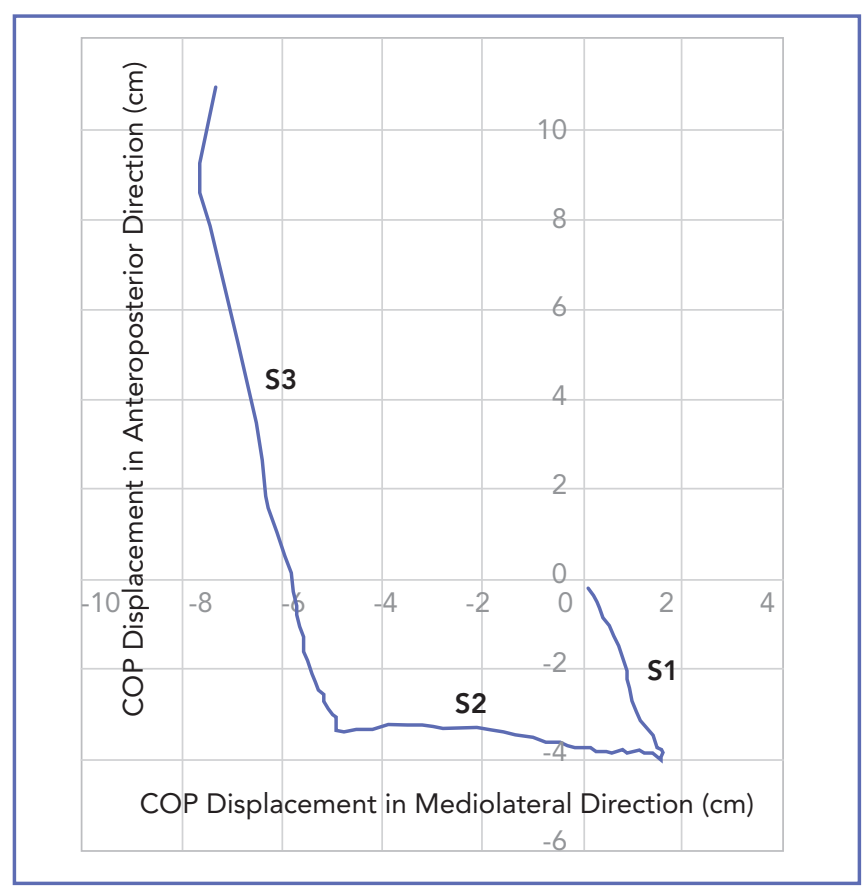

Figure 1. GI profile of an athletes with FAl before intervention (COP: Center of pressure) ending approximately at the greater trochanter. Afterward, the second I-strip was applied in the same manner starting at the anterior iliac crest (figure 2, B) (17).
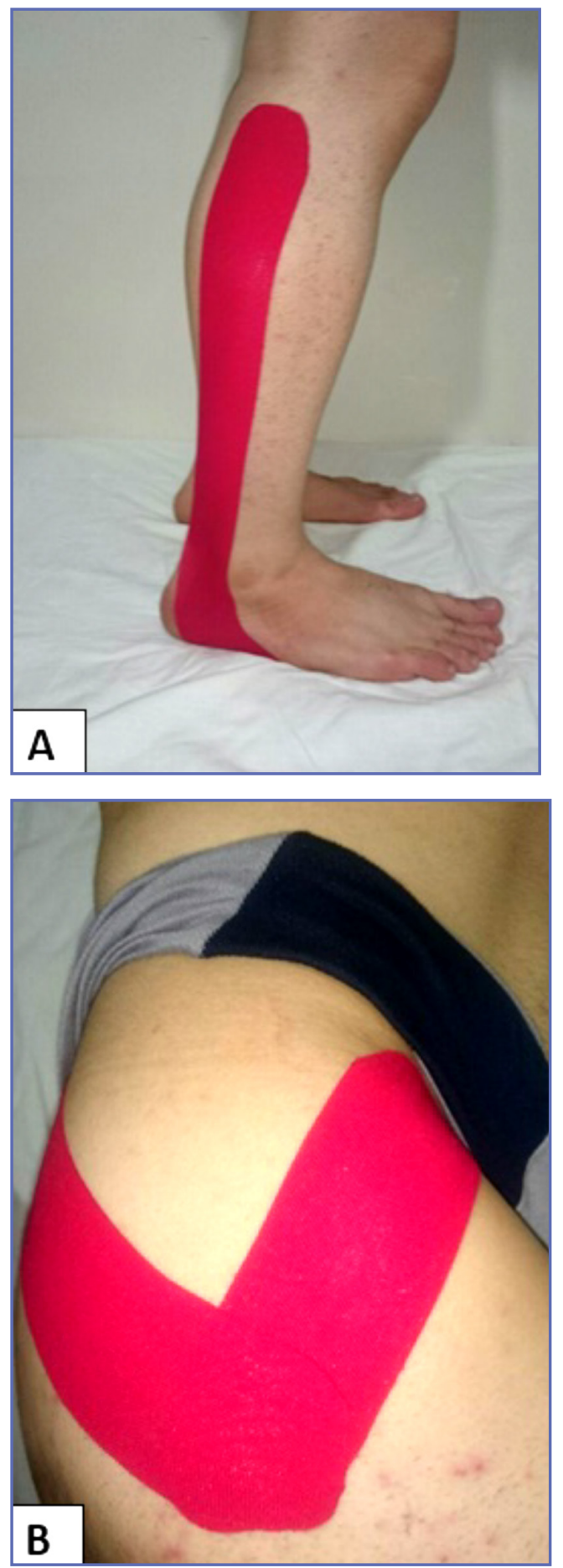

Figure 2. A: kinesio taping application on peroneus longus, B: kinesio taping application on gluteus medius

Muscles, Ligaments and Tendons Journal 2019;9 (2) 


\section{DATA REDUCTION}

The COP displacement at three phases of GI were recorded before and 30 minutes after the application of kinesio taping in all participants. The three phases of the GI profile were determined for each trial. The first phase (anticipatory: S1) started with the participant taking a response (second auditory) stimulus to start walking and finished with the COP transitioning to the most posterior and lateral position. The second phase (S2) was determined by the shift in COP towards the initial stance foot and finished when the COP transitioned to the most medial position. The third phase (S3) was differentiated by the COP moving in the anterior direction until toe off happened, identified by reaching the $100 \mathrm{~N}$ threshold. The COP displacement in the anteroposterior (AP), mediolateral (ML), and resultant planes was measured for all 3 phases of GI. Butterworth filter with a cutoff frequency of $10 \mathrm{~Hz}$ was used to filter all COP data (9) A Bertec triaxial force plate (Bertec Corp, Columbus, $\mathrm{OH}$, $90 * 90$ Series) with sampling frequency of $500 \mathrm{~Hz}$ and sensitivity of 10, was used to gather COP data. During each data collection sessions and before and after the interventions in each session, GI was assessed on both limbs. To assess GI, participants were instructed to stand with their feet shoulder width apart on a force plate (14). When standing on the force plate, the participants were asked to breathe naturally (avoid deep breathing, yawning or deliberate coughing), hang their arms around the body, and look up to the opposite wall (at a distance of about 4 meters). Participants were instructed to walk based on auditory cues.

When participants stood on the force plate, two auditory stimuli were provided at two-second intervals, one warning stimulus to prepare them and one response stimulus to start the gait (The duration of each stimulus was 100 milliseconds, the intensity was $60 \mathrm{~dB}$, and the frequency was $2 \mathrm{kHz}$ ). The sequence of these stimuli was programmed by software and synchronized by the recording of force plate data (15).

Participants were asked to walk with the tested leg in response to the response stimulus (second stimulus), so that their first step was inside the force plate and the next step outside it. The trials were repeated 3 times for each leg before and after the intervention of each session.

\section{STATISTICAL ANALYSIS}

All data were analyzed using SPSS software version 25. A one-sample Kolmogorov- Smirnov test was done to determine normal distribution of all variables $(\mathrm{P}>0.05)$. Reliability analysis for all the outcome measures was performed by ICC in a group consisting of all subjects. The mean value of all three sets of data gathering was used for final analysis, to obtain the highest possible reliable value.

Independent $\mathrm{t}$-test was employed to check the demographic variables between the athletes with FAI and the control group. Multifactorial 2 (FAI and control groups) * 2 (kinesio taped and non- kinesio taped legs) *2 (distal and proximal-distal kinesio taping) and * 2 (before and after intervention) repeated measure ANOVA were used. Each test comprised of 3 within-subject (leg, intervention, time of evaluation) and 1 between-subject (group) factors. Repeated measurement assumption was checked. When the sphericity assumption was rejected, the Greenhouse-Geisser test was applied. P-values less than 0.05 were used on all statistical tests.

\section{RESULTS}

The distribution of all variables regarding both groups was normal, according to the values of Kolmogorov-Smirnov. Fifteen male athletes with FAI (age: mean value $=23.07, \mathrm{~s}$ $=4.76$ years old, weight: mean value $=70.80, \mathrm{~s}=8.90 \mathrm{Kg}$, height: mean value $=179.53, \mathrm{~s}=4.39 \mathrm{Cm}$, BMI: mean value $=21.95, \mathrm{~s}=2.43 \mathrm{Kg} \cdot \mathrm{M}^{-2}$ ) and fifteen healthy athletes (age: mean value $=23.73, \mathrm{~s}=4.95$ years old, weight: mean value $=75.87, \mathrm{~s}=6.07 \mathrm{Kg}$, height: mean value $=177.07$, $\mathrm{s}=5.51 \mathrm{Cm}, \mathrm{BMI}$ : mean value $=24.25, \mathrm{~s}=2.27 \mathrm{Kg} . \mathrm{M}$ $\left.{ }^{2}\right)$ participated in this study. There were no significant differences between the 2 groups in demographic data ( $\mathrm{P}$ $>0.05)$ (Table I).

Table I. The results of the Independent t-test for the comparison of anthropometric data between athletes with FAI and control group ( $n=15$ in each group)

\begin{tabular}{lccccc}
\hline \multicolumn{1}{c}{ Variables } & \multicolumn{2}{c}{ Mean \pm SD } & & Range & Sig. \\
& FAI & Control & FAI & Control & \\
\hline Age $($ Year $)$ & $23.07 \pm 4.76$ & $23.73 \pm 4.95$ & $18-32$ & $18-32$ & 0.71 \\
\hline Weight $(\mathbf{K g})$ & $70.80 \pm 8.90$ & $75.87 \pm 6.07$ & $55-91$ & $64-86$ & 0.08 \\
\hline Height $(\mathbf{C m})$ & $179.53 \pm 4.39$ & $177.07 \pm 5.51$ & $171-187$ & $169-188$ & 0.19 \\
\hline BMI $\left(\mathbf{K g} / \mathbf{M}^{2}\right)$ & $21.9 \pm 2.43$ & $24.25 \pm 2.27$ & $17.36-26.88$ & $20.09-28.38$ & 0.06 \\
\hline
\end{tabular}


ICC values were interpreted according to Munro's classification of reliability: 0.26 to 0.49 reflects a low correlation, 0.50 to 0.69 reflects a moderate correlation, 0.70 to 0.89 reflects a high correlation and 0.90 to1.00 indicates a very high correlation (18). All of the variables had a moderate to high correlation, except for resultant displacement of COP in anticipatory phase after distal kinsio taping in non- kinesio taped leg (ICC $=0.45)$ of healthy athletes, anteroposterior displacement of $\mathrm{COP}$ in anticipatory phase before and after distal kinsio taping in non- kinesio taped $\operatorname{leg}(\mathrm{ICC}=0.41,0.39$, respectively) of athletes with FAI , resultant displacement of COP in anticipatory phase after distal kinsio taping in non- kinesio taped leg $(\mathrm{ICC}=0.46)$ of athletes with FAI, anteroposterior displacement of COP in weight transfer phase after distal kinesio taping in kinsio taped leg $(\mathrm{ICC}=0.39)$ of athletes with FAI, anteroposterior displacement of COP in weight transfer phase before distal kinesio taping in non- kinsio taped leg $(\mathrm{ICC}=0.41)$ of athletes with FAI, mediolateral displacement of COP in weight transfer phase after proximal- distal kinesio taping in kinsio taped leg $(\mathrm{ICC}=0.38)$ of athletes with FAI, resultant displacement of COP in weight transfer phase before distal kinsio- taping in non- kinesio taped leg (ICC= 0.43 ) of athletes with FAI, resultant displacement of COP in weight transfer phase after proximal- distal kinsio taping in kinesio taped leg $(\mathrm{ICC}=0.44)$ of athletes with FAI, anteroposterior displacement of COP in locomotive phase before distal kinsio taping in kinesio taped leg $(\mathrm{ICC}=0.41)$ of athletes with FAI, mediolateral displacement of COP in locomotive phase before distal kinesio taping in kinsio taped leg (ICC $=$ 0.46) of athletes with FAI, mediolateral displacement of $\mathrm{COP}$ in locomotive phase before proximal-distal kinesiotaping in non- kinsio-taped leg $(\mathrm{ICC}=0.46)$ of athletes with
FAI, and, resultant displacement of COP in locomotive phase before distal kinsio taping in kinesio taped leg (ICC= 0.43) of athletes with FAI.

Tables II, III, and IV show the mean and standard deviation value of the center of pressure parameters during anticipatory, weight transfer and locomotive phases of GI before and after the 2 kinesio taping interventions in the kinesio tape and non- kinesio tape legs of the athletes with FAI and healthy athletes.

According to the results of multifactorial 2 (FAI and control groups) * 2 (kinesio taped and non- kinesio taped legs) * 2 (distal and proximal-distal kinesio taping) and *2 (before and after intervention) repeated measure ANOVA, there weren't any significant result for factor effect and group effect for all of the 9 variables of the study $(\mathrm{P}>0.05)$. Also, we did not observe any significant group by factor interaction effects $(\mathrm{P}>0.05)$, indicating that each group respond to the interventions in a similar pattern.

\section{DISCUSSION}

According to the results of the multifactorial repeated measure ANOVA, none of the 3 within-subject (tested leg, intervention, time of evaluation) and the between-subject (group) factor effects and the interaction effects of them for all of the COP displacement in three phases and two directions of GI profile were not significant $(\mathrm{P}>0.05)$.

The findings of the previous studies suggest that the effective prevention and rehabilitation of ankle sprains should include attention to walking patterns and foot biomechanics in people with the risk of ankle sprains, although routine clinical evaluation after ankle sprain does not include walking analysis. It has also been shown that taping and bracing

Table II. The Center of Pressure Parameters during the Anticipatory Phase of $\mathrm{Gl}$ ( $\mathrm{n}=15$ in each group)

\begin{tabular}{|c|c|c|c|c|c|c|c|c|}
\hline \multirow{3}{*}{ Variables } & \multicolumn{4}{|c|}{ Distal kinesio taping } & \multicolumn{4}{|c|}{ Proximal- distal kinesio taping } \\
\hline & \multicolumn{2}{|c|}{ Kinesio taped leg } & \multicolumn{2}{|c|}{ Non-kinesio taped leg } & \multicolumn{2}{|c|}{ Kinesio taped leg } & \multicolumn{2}{|c|}{ Non-kinesio taped leg } \\
\hline & Before & After & Before & After & Before & After & Before & After \\
\hline \multicolumn{9}{|c|}{ FAI } \\
\hline AP Dis & $4.49 \pm 4.65$ & $3.33 \pm 1.62$ & $4.18 \pm 2.58$ & $2.84 \pm 0.95$ & $4.56 \pm 1.93$ & $4.29 \pm 4.37$ & $4.46 \pm 1.89$ & $3.23 \pm 1.34$ \\
\hline ML Dis & $1.77 \pm 1.38$ & $1.89 \pm 1.03$ & $2.38 \pm 1.63$ & $1.97 \pm 1.75$ & $1.48 \pm 0.86$ & $1.94 \pm 1.62$ & $1.54 \pm 1.17$ & $1.87 \pm 1.17$ \\
\hline Resultant Dis & $5.09 \pm 4.70$ & $4.04 \pm 1.68$ & $4.94 \pm 2.15$ & $3.98 \pm 1.23$ & $4.96 \pm 1.86$ & $5.07 \pm 4.48$ & $5.11 \pm 1.68$ & $4.11 \pm 1.35$ \\
\hline \multicolumn{9}{|c|}{ Control } \\
\hline AP Dis & $3.53 \pm 2.20$ & $5.67 \pm 6.97$ & $4.22 \pm 2.47$ & $4.62 \pm 4.00$ & $4.19 \pm 1.81$ & $3.93 \pm 2.08$ & $4.33 \pm 1.42$ & $3.42 \pm 1.17$ \\
\hline ML Dis & $1.46 \pm 1.01$ & $1.59 \pm 0.59$ & $1.62 \pm 1.01$ & $3.00 \pm 4.39$ & $1.33 \pm 0.72$ & $1.42 \pm 0.80$ & $1.39 \pm 0.83$ & $1.42 \pm 0.72$ \\
\hline Resultant Dis & $4.22 \pm 1.93$ & $6.19 \pm 6.82$ & $4.94 \pm 2.05$ & $6.38 \pm 5.13$ & $4.61 \pm 1.63$ & $4.40 \pm 2.01$ & $4.85 \pm 1.27$ & $3.92 \pm 1.21$ \\
\hline
\end{tabular}

Abbreviations: AP, Anteroposterior; ML, Mediolateral; Dis, Displacement $(\mathrm{cm})$. Data were expressed mean \pm SD. 
Table III. The Center of Pressure Parameters during the Weight Transfer Phase of GI ( $n=15$ in each group)

\begin{tabular}{|c|c|c|c|c|c|c|c|c|}
\hline \multirow{3}{*}{ Variables } & \multicolumn{4}{|c|}{ Distal kinesio taping } & \multicolumn{4}{|c|}{ Proximal- distal kinesio taping } \\
\hline & \multicolumn{2}{|c|}{ Kinesio taped leg } & \multicolumn{2}{|c|}{ Non-kinesio taped leg } & \multicolumn{2}{|c|}{ Kinesio taped leg } & \multicolumn{2}{|c|}{ Non-kinesio taped leg } \\
\hline & Before & After & Before & After & Before & After & Before & After \\
\hline \multicolumn{9}{|c|}{ FAI } \\
\hline AP Dis & $5.50 \pm 9.16$ & $2.76 \pm 1.41$ & $2.18 \pm 1.27$ & $2.09 \pm 1.58$ & $3.07 \pm 2.49$ & $5.22 \pm 4.94$ & $3.20 \pm 4.33$ & $2.43 \pm 2.01$ \\
\hline ML Dis & $6.18 \pm 14.74$ & $4.01 \pm 6.17$ & $2.88 \pm 1.48$ & $3.37 \pm 5.05$ & $1.86 \pm 1.21$ & $2.38 \pm 0.97$ & $2.67 \pm 1.54$ & $2.90 \pm 1.12$ \\
\hline Resultant Dis & $4.28 \pm 2.50$ & $5.70 \pm 6.76$ & $3.99 \pm 1.68$ & $3.43 \pm 1.57$ & $3.59 \pm 2.50$ & $6.23 \pm 4.72$ & $4.58 \pm 4.41$ & $5.04 \pm 3.21$ \\
\hline \multicolumn{9}{|c|}{ Control } \\
\hline AP Dis & $2.34 \pm 2.35$ & $2.32 \pm 1.40$ & $1.26 \pm 1.04$ & $2.44 \pm 1.46$ & $2.51 \pm 1.93$ & $2.80 \pm 2.30$ & $2.26 \pm 2.30$ & $2.90 \pm 2.72$ \\
\hline ML Dis & $2.37 \pm 1.05$ & $2.66 \pm 1.75$ & $1.87 \pm 0.96$ & $3.17 \pm 1.76$ & $2.32 \pm 1.22$ & $2.92 \pm 1.74$ & $2.77 \pm 1.26$ & $2.72 \pm 1.22$ \\
\hline Resultant Dis & $3.77 \pm 2.20$ & $3.95 \pm 1.70$ & $2.45 \pm 1.35$ & $4.48 \pm 1.82$ & $3.76 \pm 2.03$ & $4.51 \pm 2.28$ & $4.12 \pm 1.87$ & $4.61 \pm 2.40$ \\
\hline
\end{tabular}

Abbreviations: AP, Anteroposterior; ML, Mediolateral; Dis, Displacement $(\mathrm{cm})$. Data were expressed mean \pm SD.

Table IV. The Center of Pressure Parameters during the Locomotive Phase of $\mathrm{Gl}$ ( $\mathrm{n}=15$ in each group)

\begin{tabular}{|c|c|c|c|c|c|c|c|c|}
\hline \multirow{3}{*}{ Variables } & \multicolumn{4}{|c|}{ Distal kinesio taping } & \multicolumn{4}{|c|}{ Proximal- distal kinesio taping } \\
\hline & \multicolumn{2}{|c|}{ Kinesio taped leg } & \multicolumn{2}{|c|}{ Non-kinesio taped leg } & \multicolumn{2}{|c|}{ Kinesio taped leg } & \multicolumn{2}{|c|}{ Non-kinesio taped leg } \\
\hline & Before & After & Before & After & Before & After & Before & After \\
\hline \multicolumn{9}{|c|}{ FAI } \\
\hline AP Dis & $\begin{array}{l}34.05 \pm \\
10.88\end{array}$ & $\begin{array}{l}29.23 \pm \\
16.13\end{array}$ & $36.71 \pm 15.34$ & $\begin{array}{l}31.33 \pm \\
12.76\end{array}$ & $\begin{array}{l}35.87 \pm \\
16.34\end{array}$ & $\begin{array}{l}34.55 \pm \\
19.72\end{array}$ & $\begin{array}{l}29.06 \pm \\
13.68\end{array}$ & $\begin{array}{l}38.19 \pm \\
16.19\end{array}$ \\
\hline ML Dis & $7.52 \pm 2.87$ & $6.66 \pm 3.75$ & $8.91 \pm 3.88$ & $\begin{array}{l}11.18 \pm \\
13.23\end{array}$ & $7.76 \pm 6.80$ & $7.63 \pm 7.46$ & $9.71 \pm 7.82$ & $9.53 \pm 4.59$ \\
\hline Resultant Dis & $\begin{array}{l}35.16 \pm \\
11.09\end{array}$ & $\begin{array}{l}30.07 \pm \\
16.64\end{array}$ & $39.34 \pm 15.88$ & $\begin{array}{l}32.85 \pm \\
13.09\end{array}$ & $\begin{array}{l}38.44 \pm \\
16.01 \\
\end{array}$ & $\begin{array}{l}34.33 \pm \\
19.00\end{array}$ & $\begin{array}{l}32.64 \pm \\
14.97\end{array}$ & $\begin{array}{l}38.54 \\
\pm 15.70 \\
\end{array}$ \\
\hline \multicolumn{9}{|c|}{ Control } \\
\hline AP Dis & $\begin{array}{l}37.16 \pm \\
16.19\end{array}$ & $\begin{array}{l}38.49 \pm \\
15.97\end{array}$ & $41.21 \pm 14.02$ & $\begin{array}{l}37.27 \pm \\
12.54\end{array}$ & $\begin{array}{l}39.60 \pm \\
17.01\end{array}$ & $\begin{array}{l}42.25 \pm \\
17.14\end{array}$ & $\begin{array}{l}38.85 \pm \\
13.61\end{array}$ & $\begin{array}{l}38.61 \pm \\
16.53\end{array}$ \\
\hline ML Dis & $8.40 \pm 3.70$ & $10.43 \pm 9.16$ & $7.26 \pm 4.29$ & $8.00 \pm 3.32$ & $\begin{array}{l}7.42 \pm \\
4.86\end{array}$ & $9.40 \pm 4.77$ & $7.48 \pm 3.70$ & $7.66 \pm 3.10$ \\
\hline Resultant Dis & $\begin{array}{l}38.58 \pm \\
16.23\end{array}$ & $\begin{array}{l}42.54 \pm \\
16.13\end{array}$ & $42.15 \pm 14.31$ & $\begin{array}{l}38.64 \pm \\
12.68 \\
\end{array}$ & $\begin{array}{l}40.55 \pm \\
17.23\end{array}$ & $\begin{array}{l}42.82 \pm \\
16.88 \\
\end{array}$ & $\begin{array}{l}39.20 \pm \\
13.02\end{array}$ & $\begin{array}{l}39.58 \pm \\
14.43\end{array}$ \\
\hline
\end{tabular}

Abbreviations: AP, Anteroposterior; ML, Mediolateral; Dis, Displacement (cm). Data were expressed mean \pm SD.

of the ankle can reduce the occurrence of recurrent sprain (19). A few studies have been conducted in the field of walking variables as risk factors for ankle sprains.

Willems et al. (19) showed that in the group with inversion type sprain, the center of pressure in the last contact of the foot is more outward. This suggests that the force needed to invert the ankle in in these patients is less and they have less adaptive changes than healthy people when walking or running on an uneven surface. On the other hand, they did not find any difference in maximum pressure and impulses on the heels between the two groups. Becker and his colleagues measured the foot pressure distribution during walking in people with functional instability and mechanical ankle instability. They found more impulse under the external part of the heel in the ankles with functional instability but in the ankles with functional instability, no difference was observed in the heel area (20). These findings are probably due to methodological differences and the difference in the division of the heel into different areas.

The findings of the study by Hass and his colleagues explicitly state that CAI negatively affects the supera- spinal aspects of the control motor. They showed these changes by reduced magnitude of the normalized COP excursion during the S1 and S2 phases of GI. Older adults and a large 
population of patients are at risk of falling, these individuals may purposefully reduce the range of COP displacement in the posterior direction to reduce the mechanical and postural challenges during the onset of walking as well as the risk of falling. Older adults and a large population of patients are at risk of falling, these individuals may purposefully reduce the range of displacement of the center of the pressure in the posterior direction to reduce the mechanical and postural challenges during the onset of walking as well as the risk of falling. Although it seems that falling in people with CAI is not a concern, but it indicates an impaired postural control. Also despite the apparent benefits of changes seen in GI in people with CAI to maintain steady state, these people still experience giving way and recurring injury. Therefore, the supra-spinal changes observed in this study should be considered as maladaptive or ineffective changes in movement organization that limit the sensory-motor system and reduce the ability of the individual to meet the demands of the tasks (21).

Our study results were not consistent with the results of Willems et al., backer et al., and Hass et al. studies (19-21). One of the reasons for the difference in our results with the results of the first two studies could be the difference in the methodology of the studies. The methodology of study of Hass and his colleagues was similar to our study, but they used a normalized COP in which each individual's stance width is worthwhile, while in our study, the stance width for individuals was constant. In addition, the participants in the mentioned three studies were non-athletes, while in our study, there were athletes who were currently exercising and participating in the tournament. For this reason, it is possible that the neuromuscular and adaptive changes occurred in our participants followed by the first injury, and they reduced the degree of freedom of the joint in terms of the compensatory motor control strategy to maintain balance and the behavior of COP of them during different phases of gait was be close to the healthy athlete's population.

Primary results from the study done by Shields et.al showed that limited improvements in postural control were seen 24 hours after the kinesio tape application, while no immediate change was observed after application and after removal (22). The mechanism by which Kinesio tape is effective can determine whether the taping effects can be immediate or that the taping should be used for some time to achieve visible improvement. Effects of kinesio tape on proprioception occurs by increasing the stimulation of the mechanoreceptors, which leads to changes in the motor efferent responses, and may subsequently result in better joint stability. The probability of the "training effect" on the motor response following adaptation to increasing stimulation of the mechanical receptors during the night exists, which may explain why improvement was observed after 24 hours of the kinesio tape application, but not immediately after the use of it. The results of the study of Delahunt et al. were consistent with the Shields et.al study and they also did not show any significant changes in postural dynamic control after tape application (23).

The results of the study by Cotrea et al. also did not support the use of kinesio tape to improve the postural sway in people with healthy ankles. Cotrea explained that the absence of the effect of Kinesio tape was due to the fact that the evaluation was carried out after 20 minutes, and it might be important that the time should pass and then the participants be evaluated (24). In addition, the sample was made up of healthy people without muscle weakness or impaired postural sways. Thus, the space for improvement with the kinesio tape is small, since they did not have a dysfunction to be improved by the kinesio tape.

Choukou et al. concluded that Kinesio tape not only has beneficial effects on the affected foot, but also improves the stability of the unaffected foot, so this technique can be used for postural instability and to improve the performance of the ankle, while the person performs his daily duties. This study used simple and usable clinical measurements, which are the length of the step, the step duration, and the duration of the double stance and swing phases (25).

From a biomechanical point of view, the use of an elastic tape can affect joint re-position sense and proprioception. Ho et al. found that the use of kinesio tape may support muscles, ligaments, and reduce the concentration of force during the landing phase and may specifically reduce the torque of plantarflexion. The taping methods that they used, included application of tape in the basket weave pattern with application of horseshoe and reinforcing strips (26).

Kunzler et al. (27) Observed postural improvement after using the elastic tape on the Achilles tendon in non- fatigued subjects. In addition, the articles showed that these cutaneous stimulation reduces postural disturbances in young and old people following peripheral neuropathic disorders and post-stroke (28).

In a general view, we can see that our study on the effects of kinesio tape in athletes with FAI is incompatible with some studies (25-27) and consistent with others (22-24). There are several reasons for these differences in the results of the studies, including the type of tape used, the technique used, the percentage of tension used, the variables to be evaluated, the evaluated task, the time of evaluation, and ultimately the population studied.

It is possible that if we did this study in a non-athletic population, and we investigated the short-term or long-term effects of kinesio tape, or more challenging protocols such as single leg stance or jump-landing, the results would be significant. 
The main limitation of this study is that the stance width of the participant on the force plate was marked and was considered the same for all tests. A constant stance width can cause biomechanical limitations in the COP displacements. Therefore, the stance width must be selected by the person himself and in the subsequent studies, the data should be normalized to the stance width.

Future studies should assess the activity of the lower extremity muscles and also examine the central processes directly for example studies of transcranial excitation or electroencephalography and are needed to examine the analysis of the movement of the lower limb, to see what changes in the knee and ankle occur when the COP displacement increases. There may be a need for further studies to accurately determine how long tape should be worn until it is able to effect, so postural control measurements must be in the serial time point between the use of the tape and some days after its application. Future studies should focus on the effects of the tape on the sensory-motor system by examining EMG activity, motion analysis, or postural control of the fatigued ankles. In addition, it is worth considering whether the effects of kinesio tape on GI parameters depend on the length of time, the number of applications, or the extent of its tension. Before starting the new studies, individuals with FAI should be tested whether or not they have postural deficits in order to create the best condition for balance recovery after intervention.

\section{CONCLUSION}

According to the results of this study, there weren't any differences between athletes with and without FAI in regards to the GI profile and also kinesio tape intervention for muscle facilitation could not influence the COP displacement in GI. We concluded that it is better to assess the long- term effects of kinesio tape in the more challenging environment and tasks.

\section{ACKNOWLEDGEMENT}

This study was extracted from the Ph.D. Thesis of the first author in Physical Therapy Department, School of Rehabilitation, Tehran University of Medical Sciences, Tehran, Iran (IR.TUMS. MEDICINE.REC.1396.4225). The Authors would like to thank all the participants for their contribution to the study. The Authors would also like to acknowledge the assistance of the school of rehabilitation staff.

\section{Conflict of Interest}

The authors declare that they have no conflict of interest (29)

\section{REFERENCES}

1. Van Rijn RM, Willemsen SP, Verhagen AP, Koes BW, Bierma-Zeinstra SM. Explanatory variables for adult patients' self-reported recovery after acute lateral ankle sprain. Physical therapy. 2011;91(1):77-84.

2. Herb CC, Hertel J. Current concepts on the pathophysiology and management of recurrent ankle sprains and chronic ankle instability. Current Physical Medicine and Rehabilitation Reports. 2014;2(1):25-34.

3. Hupperets MD, Verhagen EA, Van Mechelen W. Effect of unsupervised home based proprioceptive training on recurrences of ankle sprain: randomised controlled trial. Bmj. 2009;339:b2684.

4. Fereydounnia S, Shadmehr A, Moghadam ST, Olyaei G, Jalaie $\mathrm{S}$. The effect of choice reaction time task on pre-landing muscle timing in athletes with and without chronic ankle instability. Muscles, Ligaments \& Tendons Journal (MLTJ). 2018;8(2).

5. Fereydounnia S, Shadmehr A, Moghadam ST, Olyaei G, Jalaie $\mathrm{S}$, Tahmasebi A. Muscle timing in injured and non-injured leg of athletes with chronic ankle instability in response to a visual stimulus during forward jumping. Journal of bodywork and movement therapies. 2016;20(1):3-9.

6. Denyer JR, Hewitt NL, Mitchell AC. Foot structure and muscle reaction time to a simulated ankle sprain. Journal of athletic training. 2013;48(3):326-30.

7. Friel K, McLean N, Myers C, Caceres M. Ipsilateral hip abductor weakness after inversion ankle sprain. Journal of athletic training. 2006;41(1):74.

8. McKeon PO, Hertel J. Systematic review of postural control and lateral ankle instability, part I: can deficits be detected with instrumented testing? Journal of athletic training. 2008;43(3):293-304.

9. Hartley EM, Hoch MC, McKeon PO. Reliability and responsiveness of gait initiation profiles in those with chronic ankle instability. Gait \& posture. 2016;49:86-9.

10. Lima BN, Lucareli PR, Gomes WA, Silva JJ, Bley AS, Hartigan $\mathrm{EH}$, et al. The acute effects of unilateral ankle plantar flexors static-stretching on postural sway and gastrocnemius muscle activity during single-leg balance tasks. Journal of sports science \& medicine. 2014;13(3):564.

11. Mostafavifar M, Wertz J, Borchers J. A systematic review of the effectiveness of kinesio taping for musculoskeletal injury. The Physician and sportsmedicine. 2012;40(4):33-40.

12. Sihlali BH, Masenyetse LJ, Maritz J, Clarke L, Swart JJ, Steenkamp L, et al. Effect of kinesio taping on explosive muscle power of gluteus maximus of male athletes. South african Journal of Sports medicine. 2012;24(3):75-80.

13. Gribble PA, Delahunt E, Bleakley C, Caulfield B, Docherty C, Fourchet F, et al. Selection criteria for patients with chronic ankle instability in controlled research: a position statement of the International Ankle Consortium. JOSPT, Inc. JOSPT, 1033 North Fairfax Street, Suite 304, Alexandria, VA 22134-1540; 2013.

14. Uemura K, Yamada M, Nagai K, Tanaka B, Mori S, Ichihashi N. Fear of falling is associated with prolonged anticipatory postural adjustment during gait initiation under dual-task conditions in older adults. Gait \& posture. 2012;35(2):282-6.

15. Fujiwara K, Tomita H, Maeda K, Kunita K. Effects of neck flexion on contingent negative variation and anticipatory postural control during arm movement while standing. Journal of Electromyography and Kinesiology. 2009;19(1):113-21. 
16. Bicici S, Karatas N, Baltaci G. Effect of athletic taping and kinesiotaping $®$ on measurements of functional performance in basketball players with chronic inversion ankle sprains. International journal of sports physical therapy. 2012;7(2):154.

17. Miller J, Westrick R, Diebal A, Marks C, Gerber JP. Immediate effects of lumbopelvic manipulation and lateral gluteal kinesio taping on unilateral patellofemoral pain syndrome: a pilot study. Sports health. 2013;5(3):214-9.

18. Carter R, Lubinsky J. Rehabilitation Research-E-Book: Principles and Applications: Elsevier Health Sciences; 2015.

19. Willems T, Witvrouw E, Delbaere K, De Cock A, De Clercq D. Relationship between gait biomechanics and inversion sprains: a prospective study of risk factors. Gait \& posture. 2005;21(4):379-87.

20. Becker H, Rosenbaum D, Claes L, Gerngroß H. Dynamische Pedographie zur Abklärung der funktionellen Sprunggelenkinstabilität. Der Unfallchirurg. 1997;100(2):133-9.

21. Hass CJ, Bishop MD, Doidge D, Wikstrom EA. Chronic ankle instability alters central organization of movement. The American journal of sports medicine. 2010;38(4):829-34.

22. Shields C. The effects of Kinesio Taping $₫$ on postural control deficits in healthy ankles, copers, and individuals with functional ankle instability: University of Delaware; 2012.

23. Delahunt E, McGrath A, Doran N, Coughlan GF. Effect of taping on actual and perceived dynamic postural stability in persons with chronic ankle instability. Archives of physical medicine and rehabilitation. 2010;91(9):1383-9.

24. Correia C, Lopes S, Gonçalves R, Torres R, Pinho F, Gonçalves $\mathrm{P}$, et al. Kinesiology taping does not change fibularis longus latency time and postural sway. Journal of bodywork and movement therapies. 2016;20(1):132-8.

25. Choukou M-A, Hijazi S. Effectiveness of ankle taping on ankle joint kinematics during walking on level ground. Foot \& ankle specialist. 2013;6(5):352-5.

26. Ho Y-H, Lin C-F, Chang C-H, Wu H-W. Effect of ankle kinesio taping on vertical jump with run-up and countermovement jump in athletes with ankle functional instability. Journal of physical therapy science. 2015;27(7):2087-90.

27. Kunzler MR, Lopes LM, Ueda LS, de Britto MA, Carpes FP. Does skin stimulation compensate impairments in postural control after ankle plantar flexors fatigue? Gait \& posture. 2013;37(4):611-4.

28. Abdi E, Ferrari F, Choukou M, De Ru E, Boyer F, Trenchard $\mathrm{H}$, et al. Does "Kinesio-Taping" influence dynamic standing balance? International Journal Series in Multidisciplinary Research (IJSMR)(ISSN: 2455-2461). 2016;2(1):1-10.

29. Padulo J, Oliva F, Frizziero A, Maffulli N. Muscles, Ligaments and Tendons Journal - Basic principles and recommendations in clinical and field Science Research: 2018 update. MLTJ 2018; 8(3): 305 - 307. 